\title{
Successful Treatment of Pancreatic Fistula Following Surgery for Congenital Biliary Dilatation with Endoscopic Ultrasound-Guided Transduodenal Drainage
}

\author{
Shigemasa Suzuki ${ }^{a}$ Norio Kubo ${ }^{a}$ Nobuhiro Hosoi ${ }^{a}$ Takashi Ooki ${ }^{a}$ \\ Naoki Matsumura ${ }^{a}$ Ryusuke Aihara ${ }^{a}$ Akira Mogi ${ }^{a}$ Yasuo Hosouchi ${ }^{a}$ \\ Yasuji Nishida ${ }^{a}{\text { Takeshi Hatanakab }{ }^{b} \text { Yoshiki Tanakab Hiroshi Saekic }}^{\mathrm{c}}$ \\ Ken Shirabe ${ }^{c}$ \\ aDepartment of Surgery, Gunma Prefecture Saiseikai Maebashi Hospital, Maebashi, \\ Japan; 'bepartment of Gastroenterology, Gunma Prefecture Saiseikai Maebashi Hospital, \\ Maebashi, Japan; 'Department of General Surgical Science, Graduate School of Medicine, \\ Gunma University, Maebashi, Japan
}

\section{Keywords}

Pancreatic fistula . Endoscopic ultrasound-guided transduodenal drainage $\cdot$ Congenital biliary duct dilation

\begin{abstract}
Despite improvements in surgical techniques and perioperative management, postoperative pancreatic fistula (PF) is often difficult to treat and can be fatal due to various complications without effective drainage. Here, we report a case of PF following surgery for congenital biliary dilatation (CBD) successfully managed by endoscopic ultrasound (EUS)-guided transduodenal drainage. A 55-year-old woman underwent extrahepatic bile duct resection, including the gallbladder, and biliary tract reconstruction for CBD. On the 10th postoperative day (POD), computed tomography $(\mathrm{CT})$ showed fluid retention observed from the upper edge of the pancreatic head to the surface of the right lobe of the liver. First, percutaneous fine-needle aspiration was performed on the fluid retention in the lateral part of the liver on the 11th POD. The amylase level in the drainage was high $(30,156 \mathrm{U} / \mathrm{L})$, and we diagnosed it as PF. Percutaneous drainage was difficult for fluid retention on the cut surface of the pancreas; thus, drainage under EUS guidance was decided. On the 13th POD, EUS was performed, a scan of the
\end{abstract}


duodenal bulb revealed fluid retention with debris inside, and approximately $20-\mathrm{mL}$ fluid was aspirated (amylase: 139,200 U/L). Although the inflammatory response temporarily improved, it recurred, so we decided to perform continuous drainage. On the 21st POD, EUS was performed again; a 19-G needle was used; a 0.025-in angle-type Jagwire was advanced into the fluid retention and expanded using a 7-Fr dilator; and then, a 6-Fr endoscopic nasoabscess drain (ENAD) tube was placed. On the 29th POD, CT showed that the fluid retention on the upper edge of the head of the pancreas had shrunk to a thickness of approximately $20 \mathrm{~mm}$. On the 30th POD, the patient started eating. The ENAD tube was removed on the 38th POD. The patient was discharged from the hospital on the 45th POD without any symptoms. EUSguided transduodenal drainage is an effective treatment option for postoperative PF following surgery for CBD.

\section{Introduction}

Postoperative pancreatic fistula (PF) mainly occurs due to insufficiency of suturing of the anastomotic site after pancreatectomy and leakage of pancreatic juice from the branched pancreatic duct exposed in the cross-section. Once a PF develops, it is often refractory and, without effective drainage, can be accompanied by bacterial infections, intra-abdominal abscess, sepsis, and even pseudoaneurysm formation and rupture, leading to intra-abdominal bleeding, which can be fatal [1]. Although, recently, endoscopic ultrasound (EUS)-guided transmural drainage for PF after pancreaticoduodenectomy has been reported to be useful [2], few authors have reported EUS-guided transmural drainage after surgery for congenital biliary dilatation (CBD). Here, we report a case of postoperative PF following surgery for CBD successfully managed by EUS-guided transduodenal drainage.

\section{Case Presentation}

A 55-year-old woman underwent extrahepatic bile duct resection, including the gallbladder, and biliary tract reconstruction for Todani classification type IV-A CBD (Fig. 1a, b). The reconstruction method used was hepaticojejunostomy with Roux-en-Y. The drain was placed in the foramen of Winslow, dorsal of the anastomotic site. The patient started eating foods on the 5th postoperative day (POD). No problems with the drainage were encountered, and it was removed on the 7th POD. However, the inflammatory response was prolonged after that. On the 10th POD, computed tomography (CT) showed fluid retention observed from the upper edge of the pancreatic head (the site where the bile duct was resected) to the surface of the right lobe of the liver (Fig. 2a, b). First, percutaneous fine-needle aspiration was performed on the fluid retention in the lateral part of the liver on the 11th POD. The amylase level in the drainage was high (i.e., 30,156 U/L), and we diagnosed it as PF (grade B according to the definition by the International Study Group of PF) [3]. Percutaneous drainage was difficult to perform for fluid retention on the cut surface of the pancreas, so drainage under EUS guidance was decided. On the 13th POD, EUS was performed, a scan of the duodenal bulb revealed fluid retention with debris inside (Fig. 3a), and approximately 20 -mL fluid was aspirated (Fig. 3). The amylase level in the drainage was also high $(139,200 \mathrm{U} / \mathrm{L})$. On the 14 th POD, magnetic resonance cholangiopancreatography was performed, and no damage to the main pancreatic duct was observed. We determined that it was a PF from the exfoliated surface of the pancreas. Although the inflammatory response temporarily improved, it recurred,

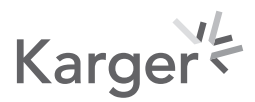



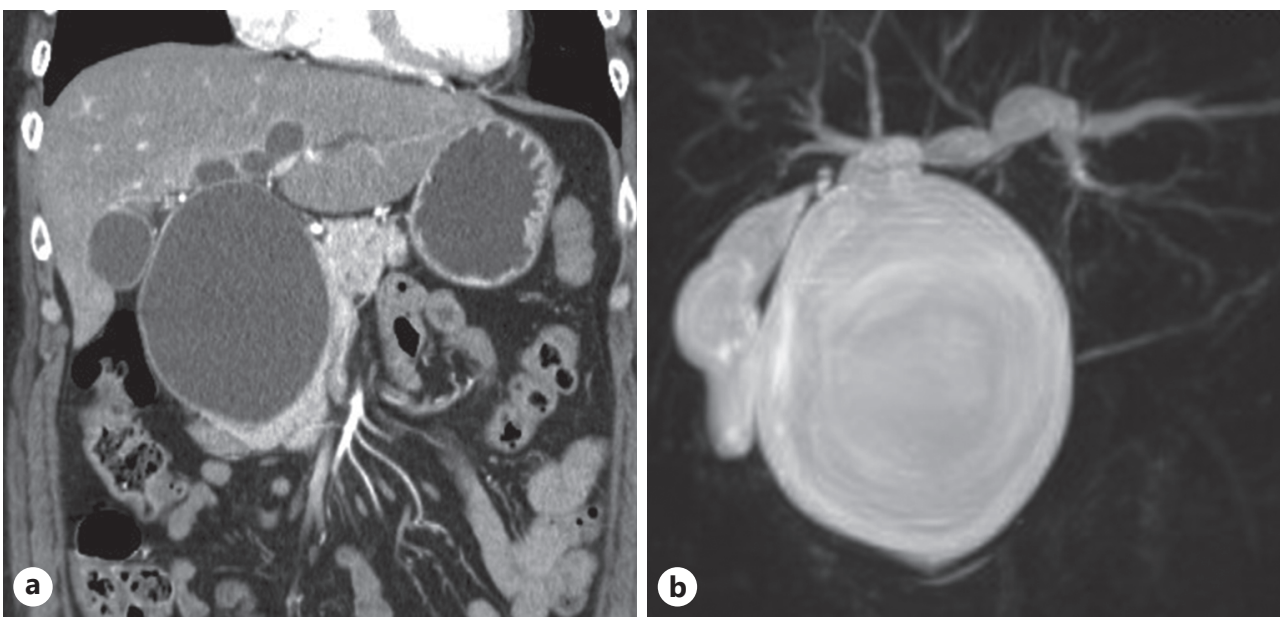

Fig. 1. Preoperative image. a CT showed a marked cystic dilation of the entire common bile duct (maximum diameter: $81.5 \mathrm{~mm}$ ) and an intrahepatic bile duct dilated near the hilum. b Magnetic resonance cholangiopancreatography showed dilation of the common bile duct and intrahepatic bile duct. Pancreatobiliary maljunction (bile duct type) was also observed, diagnosed as Todani classification type IV-A.
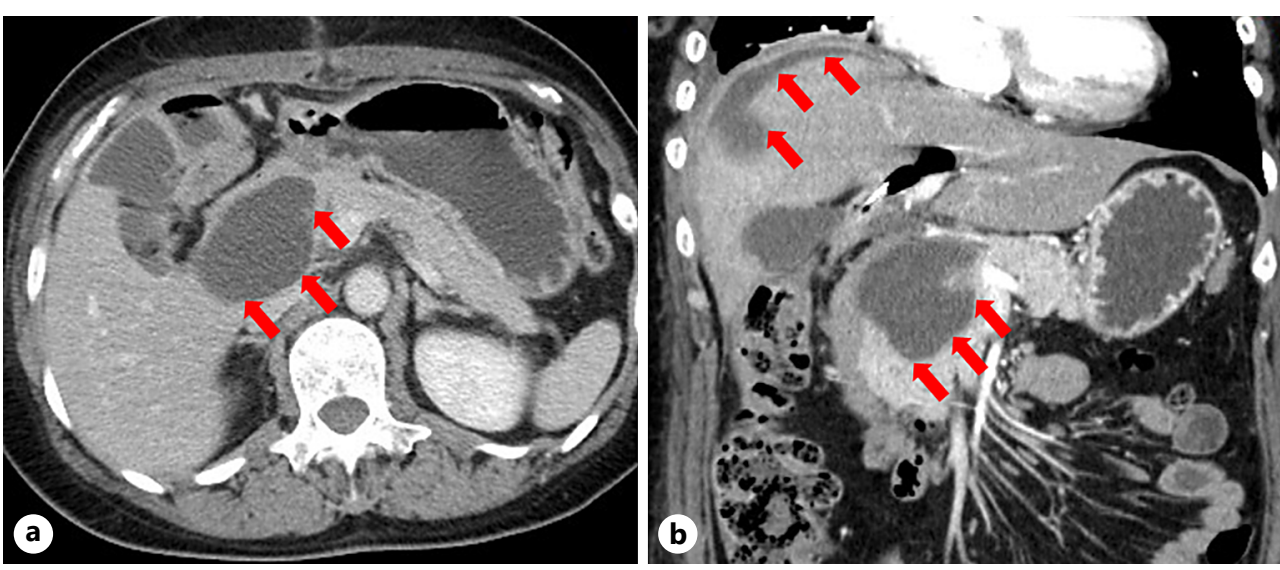

Fig. 2. CT on the 10th POD revealed PF. Fluid retention with a maximum diameter of $58 \mathrm{~mm}$ near the upper edge of the head of the pancreas and the anterior surface of the right lobe of the liver is observed (arrow). a Axial section. b Coronal section.

so we decided to perform continuous drainage. On the 21st POD, EUS was performed again, showing that the bulb was swollen due to fluid retention in the PF. A 19-G needle was punctured; a 0.025-in angle-type Jagwire was advanced into the fluid retention (Fig. 3c) and expanded using a 7-Fr dilator; and then, a 6-Fr endoscopic nasoabscess drain (ENAD) tube was placed (Fig. 3d). Approximately 50-mL purulent drainage was observed. On the 29th POD, CT showed that the fluid retention on the upper edge of the head of the pancreas had shrunk to a thickness of approximately $20 \mathrm{~mm}$ (Fig. 4a). On the 30th POD, the patient started eating foods. On the 31st POD, contrast enhancement using urografin was performed from the ENAD tube. It was imaged along the shape of the pigtail loop. The tube was pulled out a little and refixed. After that, almost no drainage was observed, and inflammatory reaction also improved. On the 38th POD, the ENAD tube was removed. The patient was discharged from the hospital on the 45th POD without any symptoms (Fig. 4b). 

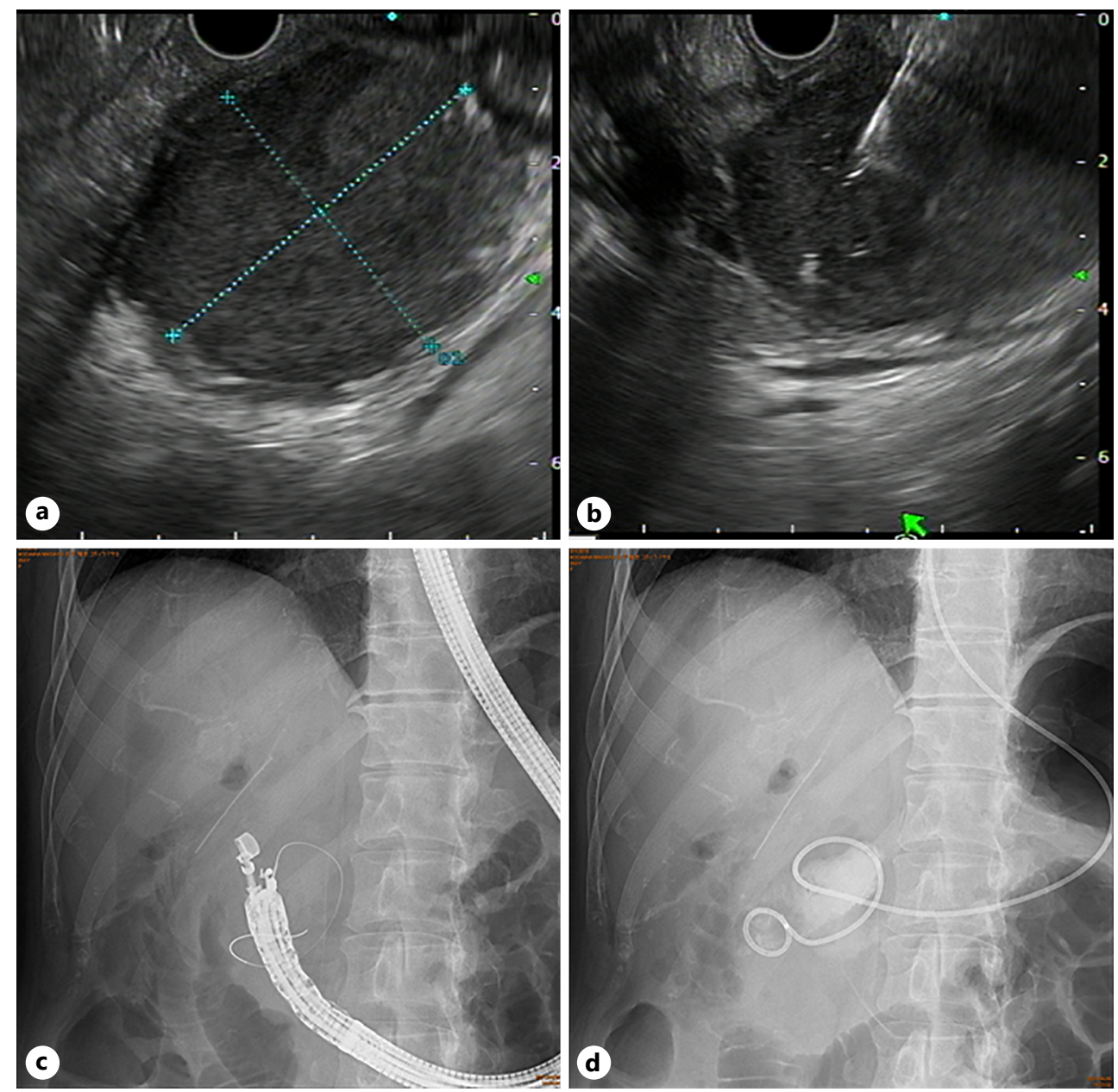

Fig. 3. Endoscopic ultrasound-guided transduodenal drainage. a A scan of the duodenal bulb revealed fluid retention with debris inside. b A 19-G needle was punctured for fluid drainage. c A 0.025-in angle-type Jagwire was advanced into the fluid retention site. d A 6-Fr ENAD tube was placed.
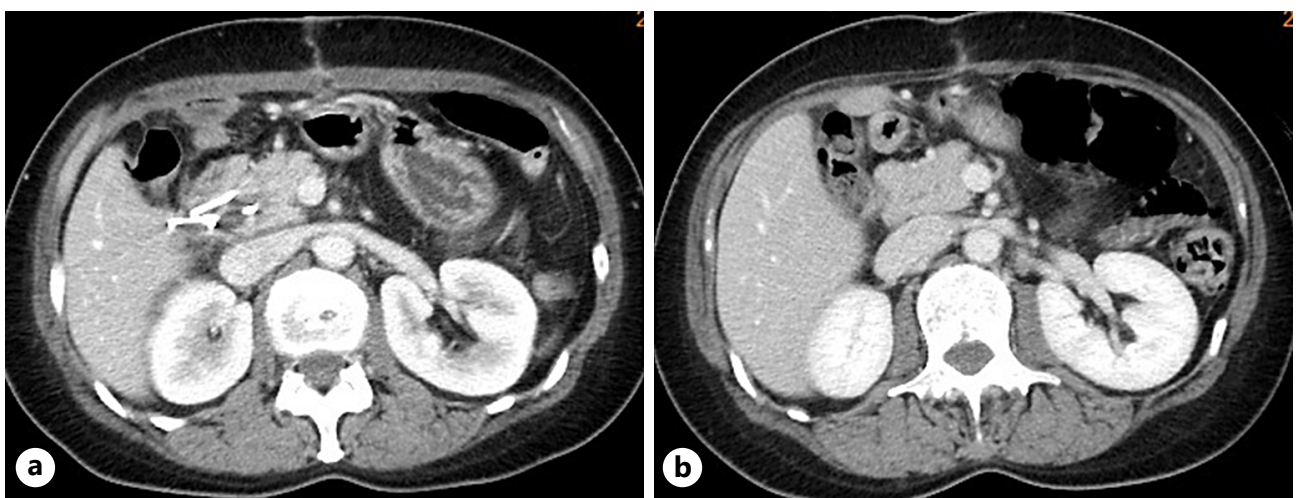

Fig. 4. CT after endoscopic ultrasound-guided transduodenal drainage. a CT on the 29th POD. The fluid retention on the upper edge of the head of the pancreas had shrunk to a thickness of approximately $20 \mathrm{~mm}$. The fluid on the surface of the liver was also reduced. $\mathbf{b}$ CT approximately 2 months after discharge. The fluid retention on the upper edge of the pancreas has disappeared. 


\section{Discussion}

CBD is a combination of localized dilatation of the extrahepatic bile duct, including the common bile duct, and anomalies of the pancreaticobiliary junction. It is a congenital dysplasia causing various pathological conditions, such as impaired outflow or reflux of bile and pancreatic juice and a complication of biliary tract cancer $[4,5]$. Since biliary tract cancer occurs at a high rate in the dilated bile duct and gallbladder, extrahepatic bile duct resection including the gallbladder, which is the carcinogenic origin, and biliary tract reconstruction are standard surgical procedures [6]. Moreover, carcinogenesis, pancreatitis, and pancreatic stones, among others, from the residual bile duct in the pancreas have been reported after surgery [7]. From this, excising the lower part of the common bile duct near the confluence of the pancreatic duct is necessary without sparing the intrapancreatic bile duct as much as possible [8].

Early complications after surgery for CBD include bleeding, PF, acute pancreatitis, gastrointestinal bleeding, and ileus; however, the frequency of these complications is not high. Todani et al. [9] have reported that 12 of 74 cases (16.4\%) had early complications, such as suture failure, bleeding, acute pancreatitis, ileus, gastrointestinal bleeding, and PF. However, in patients with a significantly large bile duct dilation as in the patient in this case report, the risk of PF is high because the detached area from the pancreas inevitably becomes large.

$\mathrm{PF}$ remains one of the most notable clinical complications that are directly linked to surgery-related death. Regarding the treatment of PF, antibacterial drugs [10], nutritional support [11], somatostatin analogs [12,13], interventional radiology [14, 15], and surgical treatment $[16,17]$, among others, were mentioned. Although the standard treatment has not yet been established, there seems to be no dispute that drainage is the most important. In the patient in this case report, a drain was placed in the foramen of Winslow. Recently, in drain management after pancreatic resection, removing the drain within 5 days is recommended if there is no suspicion of PF $[13,18]$, and even in this case, it is removed relatively early. However, it is possible that the drainage was inadequate. The drain may have been placed so that proper drainage would work for the "dent" that was hollowed out of the intrapancreatic bile duct; however, repositioning the drain is anatomically difficult.

In contrast, endoscopic drainage has been attempted for postoperative $\mathrm{PF}$, and its usefulness has been reported recently [19-21]. There are two types of endoscopic drainage: trans-papillary drainage and EUS-guided transluminal drainage. Both selections consider the PF condition. Trans-papillary drainage should be prioritized for acute peripancreatic fluid collection before cyst wall formation or with main pancreatic duct stenosis, and EUS-guided transluminal drainage should be selected if this is ineffective or if there is an infectious complication $[22,23]$. However, the aim of the trans-papillary drainage is to ensure the antegrade trans-papillary outflow of pancreatic juice and to reduce leakage of pancreatic juice secondarily. That is, the drainage effect of the stored liquid itself is not strong. In this case, there was no stenosis of the main pancreatic duct, which was accompanied by an infection; thus, EUSguided transluminal drainage was selected. Furthermore, ENAD was selected as the method of continuous drainage because priority was given to being able to observe the amount of drainage and properties in real time. It is also possible to perform cleaning and contrast. Although the patient felt discomfort in the pharynx, the discomfort was alleviated using a relatively thin tube, and eating food was possible, even during indwelling.

Considering that the indication for walled-off necrosis after acute pancreatitis is 4 weeks after the onset, there is an opinion that it is better to wait 4 weeks after the operation for the EUS-guided drainage [24]. In contrast, after pancreatic resection, adhesion of serosa of surrounding organs is observed for approximately 2-3 days [25]. The PF generated at the surgical site is expected to be localized by being surrounded by the serosa of the surrounding organs that have adhered. Therefore, unlike the case of pseudopancreatic cysts occurring in 
acute pancreatitis, hesitating to perform drainage early may not be necessary. In this case, only EUS-guided aspiration was initially performed; however, continuous drainage may have been performed from the beginning.

The purpose of EUS-guided drainage is to reduce fluid retention, and confirming the reduction of the lesion diameter of the PF by imaging examination and observing the improvement of the patient's subjective symptoms and inflammatory reaction over time are necessary. Furthermore, some complications after drainage are serious, such as bleeding and perforation. In addition to strict follow-up, if complications appear, a prompt response, including surgical procedures, is required.

In conclusion, the risk of PF is relatively high in CBD with remarkable large bile duct dilatation, similar to the patient in this case report. Although effective drainage placement during surgery seems to be the most important, EUS-guided transduodenal drainage is an effective treatment option for PF after surgery for CBD.

\section{Acknowledgment}

The authors would like to thank Enago (www.enago.jp) for the English language review.

\section{Statement of Ethics}

This study protocol was reviewed and approved by the Ethics Committee of Gunma Prefecture Saiseikai Maebashi Hospital, approval No. 2021-022. Written informed consent was obtained from the patient for publication of this case report and any accompanying images.

\section{Conflict of Interest Statement}

The authors declare no conflict of interest.

\section{Funding Sources}

No grant support or funding from public institutions or private enterprises was received for this case report.

\section{Author Contributions}

S.S. reported the case and wrote the manuscript. N.K., N.H., T.O., N.M., R.A., A.M., Y.H., T.H., and Y.T. were involved in treating the patient. Y.N., H.S., and K.S. participated in critically revising the manuscript. All authors declare that they contributed to this article and that they have read and approved the final version of the manuscript.

\section{Data Availability Statement}

All data generated or analyzed during this study are included in this article. Further inquiries can be directed to the corresponding author. 


\section{References}

1 Pedrazzoli S, Liessi G, Pasquali C, Ragazzi R, Berselli M, Sperti C. Postoperative pancreatic fistulas: preventing severe complications and reducing reoperation and mortality rate. Ann Surg. 2009 Jan;249(1):97-104.

2 Futagawa Y, Imazu H, Mori N, Kanazawa K, Chiba M, Furukawa K, et al. The effectiveness and feasibility of endoscopic ultrasound-guided transgastric drainage of postoperative fluid collections early after pancreatic surgery. Surg Laparosc Endosc Percutan Tech. 2017 Aug;27(4):267-72.

3 Bassi C, Marchegiani G, Dervenis C, Sarr M, Abu Hilal M, Adham M, et al. The 2016 update of the International Study Group (ISGPS) definition and grading of postoperative pancreatic fistula: 11 years after. Surgery. 2017 Mar;161(3):584-91.

4 Ishibashi H, Shimada M, Kamisawa T, Fujii H, Hamada Y, Kubota M, et al. Japanese clinical practice guidelines for congenital biliary dilatation. J Hepatobiliary Pancreat Sci. 2017 Jan;24(1):1-16.

5 Todani T, Watanabe Y, Narusue M, Tabuchi K, Okajima K. Congenital bile duct cysts: classification, operative procedures, and review of thirty-seven cases including cancer arising from choledochal cyst. Am J Surg. 1977 Aug;134(2):263-9.

6 Ando H, Kaneko K, Ito T, Watanabe Y, Seo T, Harada T, et al. Complete excision of the intrapancreatic portion of choledochal cysts. J Am Coll Surg. 1996 Oct;183(4):317-21.

7 Yoshikawa K, Yoshida K, Shirai Y, Sato N, Kashima Y, Coutinho DS, et al. A case of carcinoma arising in the intrapancreatic terminal choledochus 12 years after primary excision of a giant choledochal cyst. Am J Gastroenterol. 1986 May;81(5):378-84.

8 Urushihara N, Fukumoto K, Fukuzawa H, Mitsunaga M, Watanabe K, Aoba T, et al. Long-term outcomes after excision of choledochal cysts in a single institution: operative procedures and late complications. J Pediatr Surg. 2012 Dec;47(12):2169-74.

9 Todani T, Watanabe Y, Toki A, Urushihara N, Sato Y. Reoperation for congenital choledochal cyst. Ann Surg. 1988 Feb;207(2):142-7.

10 Macedo FIB, Mowzoon M, Parikh J, Sathyanarayana SA, Jacobs MJ. Disparities in the management and prophylaxis of surgical site infection and pancreatic fistula after pancreatoduodenectomy. J Hepatobiliary Pancreat Sci. 2017 May;24(5):268-80.

11 Klek S, Sierzega M, Turczynowski L, Szybinski P, Szczepanek K, Kulig J. Enteral and parenteral nutrition in the conservative treatment of pancreatic fistula: a randomized clinical trial. Gastroenterology. 2011 Jul;141(1):157-1.

12 Allen PJ, Gönen M, Brennan MF, Bucknor AA, Robinson LM, Pappas MM, et al. Pasireotide for postoperative pancreatic fistula. N Engl J Med. 2014 Aug 28;370(9):2014-22.

13 Adachi T, Kuroki T, Kitasato A, Hirabaru M, Matsushima H, Soyama A, et al. Safety and efficacy of early drain removal and triple-drug therapy to prevent pancreatic fistula after distal pancreatectomy. Pancreatology. 2015 Jul-Aug;15(4):411-6.

14 Munoz-Bongrand N, Sauvanet A, Denys A, Sibert A, Vilgrain V, Belghiti J. Conservative management of pancreatic fistula after pancreaticoduodenectomy with pancreaticogastrostomy. J Am Coll Surg. 2004 Aug;199(2):198-203.

15 Tol JA, Busch OR, van Delden OM, van Lienden KP, van Gulik TM, Gouma DJ. Shifting role of operative and nonoperative interventions in managing complications after pancreatoduodenectomy: what is the preferred intervention? Surgery. 2014 Sep;156(3):622-31.

16 Fuks D, Piessen G, Huet E, Tavernier M, Zerbib P, Michot F, et al. Life-threatening postoperative pancreatic fistula (grade C) after pancreaticoduodenectomy: incidence, prognosis, and risk factors. Am J Surg. 2009 Jun; 197(6):702-9.

17 Smits FJ, van Santvoort HC, Besselink MG, Batenburg MCT, Slooff RAE, Boerma D, et al. Management of severe pancreatic fistula after pancreatoduodenectomy. JAMA surgery. 2017 Jun 1;152(6):540-8.

18 Villafane-Ferriol N, Shah RM, Mohammed S, Van Buren G 2nd, Barakat O, Massarweh NN, et al. Evidence-based management of drains following pancreatic resection: a systematic review. Pancreas. 2018 Jan;47(1):12-7.

19 Seewald S, Groth S, Omar S, Imazu H, Seitz U, de Weerth A, et al. Aggressive endoscopic therapy for pancreatic necrosis and pancreatic abscess: a new safe and effective treatment algorithm (videos). Gastrointest Endosc. 2005 Jul;62(1):92-100.

20 Varadarajulu S, Wilcox CM, Christein JD. EUS-guided therapy for management of peripancreatic fluid collections after distal pancreatectomy in 20 consecutive patients. Gastrointest Endosc. 2011 Aug;74(2):418-23.

21 Kwon YM, Gerdes H, Schattner MA, Brown KT, Covey AM, Getrajdman GI, et al. Management of peripancreatic fluid collections following partial pancreatectomy: a comparison of percutaneous versus EUS-guided drainage. Surg Endosc. 2013 Jul;27(7):2422-7.

22 Tilara A, Gerdes H, Allen P, Jarnagin W, Kingham P, Fong Y, et al. Endoscopic ultrasound-guided transmural drainage of postoperative pancreatic collections. J Am Coll Surg. 2014 Jan;218(1):33-40.

23 Saftoiu A, Vilmann A, Vilmann P. Endoscopic ultrasound-guided drainage of pancreatic pseudocysts. Endosc Ultrasound. 2015 Oct-Dec;4(4):319-23.

24 Arvanitakis M, Dumonceau JM, Albert J, Badaoui A, Bali MA, Barthet M, et al. Endoscopic management of acute necrotizing pancreatitis: European Society of Gastrointestinal Endoscopy (ESGE) evidence-based multidisciplinary guidelines. Endoscopy. 2018 May;50(5):524-46.

25 Azeem N, Baron TH, Topazian MD, Zhong N, Fleming CJ, Kendrick ML. Outcomes of endoscopic and percutaneous drainage of pancreatic fluid collections arising after pancreatic tail resection. J Am Coll Surg. 2012 Aug; 215(2):177-85. 